\title{
CDMA based Data Transmission Methods between Vehicles
}

\author{
SP.Chokkalingam, S. Jabeer Basha, Indla Venkatesh, Yadla Srinivas
}

\begin{abstract}
There are large amounts of cars passing through the streets every day and this increases significantly in the morning or evening rush hours. The automotive industry has evolved greatly and many technologies have been introduced to automate many of its functions. The code division multiple access(CDMA) is mostly used in our daily lives. The emergence of driverless vehicles has led to a boom in the world of automotive technology, because of its importance in creating a new environment and new concepts aimed at human conformability and increased productivity. Many solutions have been appeared to offer suitable self-driving vehicles environment that is capable to overcome the arising problems. When the self-driving vehicles are well equipped, the only remaining goal is how to control external communications with other vehicles, with updates that can appear in the road and with main center. It come through by the help of CDMA network connection. This paper proposed code division multiple access as a communication technology to overcome the problems may appeared in time division multiple access. The proposed system of robot cars is implemented through high way environment scenario. The communication system is implemented via code division multiple access (CDMA) to realize high security, high quality of services, robustness and high capacity.
\end{abstract}

Keywords: Automation, Car, CDMA, Security.

\section{INTRODUCTION} stages before reaching the form we know today. The steam engine was invented by the French inventor Nicolas Joseph Cuneo in 1769. Electric cars began to appear after the invention of Hungarian AnoisGedlik model of a small car powered by electricity in 1828 . The modern cars that we use today have developed very significantly because of the great industrial development that has entered the car industry. The technology and the smart systems have also entered the industry. Modern cars have touch screens, voice commands, with a number of functions through the mobile phone, in addition to the modern cars to replace the key with the start button, and became the automotive systems rely on the electrical system helps in its work, and not rely entirely on fuel, and thus help to provide the fuel consumption will be significantly reduced.

Revised Manuscript Received on July 13, 2019.

SP.Chokkalingam, Professor, Saveetha School of Engineering, SIMATS, Chennai.

S. Jabeer Basha, UG Scholar, Saveetha School of Engineering, SIMATS, Chennai.

Indla Venkatesh, UG Scholar, Saveetha School of Engineering, SIMATS, Chennai.

Yadla Srinivas, UG Scholar, Saveetha School of Engineering, SIMATS, Chennai.
The automotive industry has undergone a number of

Roads have always been owned and operated by governments, vehicles have been largely owned and maintained by individuals, and the use of road space has not generally been based on a user payment model. The future could challenge this model, since the vehicles can belong to a small number of important operators who charge their travel costs to their customers, thus taking advantages of a previously unassigned revenue stream. The World's Road Safety Report 2015, which reflects information from 180 countries, indicated that the total number of deaths in road accidents has reached 1.25 million per year, and that the road mortality rates are the highest in low income developing countries.

Most of the accidents that lead to death are due to safety belts, drunk driving, high speed, motorcycle helmets and safety systems for children, this means that the main cause of accidents is human. Therefore, resorting to the adoption of many technical methods to control these causes and reduce them as possible. The proposed system is a flexible communication system that is able to communicate between moving license vehicles and to main centre considering any obstacle may appear in the environment.

Communication system design based on CDMA technique to be more flexible and more secure comparing with TDMA. There is a basic structure of communication infrastructure in driverless cars with CDMA.

\section{LITERATURE REVIEW}

Autonomous vehicles are heavily based on their communication systems which are internal/external system to sense surrounding environment to moving from point to others. In this case, researchers are considered communication system plays a vital role in developing and spreading self-driving vehicles [1-2]. However, some projectors seek to improve current external communication system of self-driving cars. Moreover, performance of autonomous vehicles is enhanced by improving their external communication system as well as employing some new techniques such as, artificial intelligent [3-4].

Some recent related works has been explained as below. BassemMokhtar et al. 2015 explained the VANET network and the protection constraints to obtain the obtainability of universal connectivity, protect infrastructures and reputation organization schemes that affect trust in cooperation and negotiation between mobile network units. Then they analyse VANET protection aspects, challenges and attacks, and category VANET security introducers due to various layers of network [5-7]. 
Ribal F. Atallah et al. 2015.concentrated on the latest developments in each of the research areas which has been mentioned above and highlights the open questions that remain unresolved in each one of them [8]. Then the researchers were also involved in the design of programming policies to increase the efficiency of spectrum access and optimize the performance of vehicle networks based on several conventional metrics [9].

Raffaele Bruno et al. -2015. interested in the utilise of cars as components of a multimedia sensor structure capable of capturing snapshots of the camera on the roads to support traffic observing and urban surveillance tasks. They used submodular optimization methods to build robust and efficient information gathering systems for vehicle multimedia sensor networks [10]. They explored an unconventional method to information collection that works on longer period measures and depend on solely on localized decisions instead of centralized calculations.

Susana Sousa -2017. presented a VANET agnostic architecture that allows the use of multiple communication technologies in an open and modular framework, which is an adaptation of the current standards approach, to be implemented in intelligent transport systems as a way to overcome its main limitations [11].

TesnimMekki et al. -2017.studied the model of the vehicular cloud in which focused on its characteristics and architectures. They present a description of the motivation of the vehicular cloud. Then they explored the challenges of the design. In addition, they highlighted the characteristics of cloud architectures of existing vehicles. They provided a taxonomy of vehicle clouds followed by classification criteria [12].

Gongjun Yan et al. -2017. analyzed the wireless connectivity between vehicles using mathematical models. They considered the consequence of distance, association time, acceleration, the comparative speed of cars, the range and size of messages / data in short-range car to car transportations. The numerical results in the simulations authenticate the analysis.

Communication systems play an important issue in developing and implementing self-driving vehicles. Most of the related works are concentrated on improving internal and external communication systems of self-driving vehicles [13].

\section{PROPOSED METHODOLOGY}

External/internal communication systems are considered a backbone for any autonomous system without it cannot do anything. Moreover, all fundamental operations of autonomous vehicles are based on communication systems as shown in the Fig.1[14]

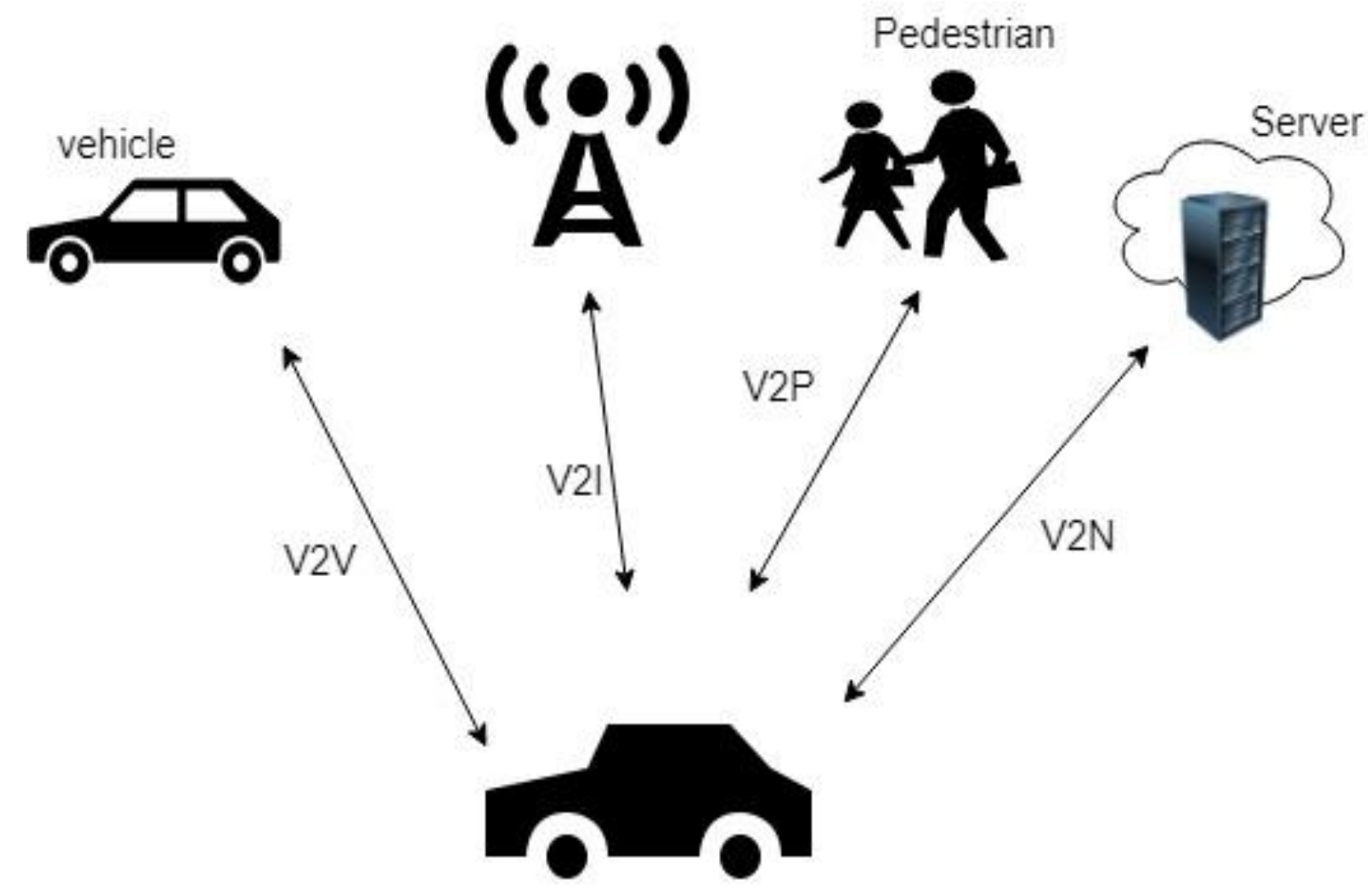

Fig.1 Vehicle to Vehicle communication

Research methodology of novel communication system is explained in this subsection. Fig.2 


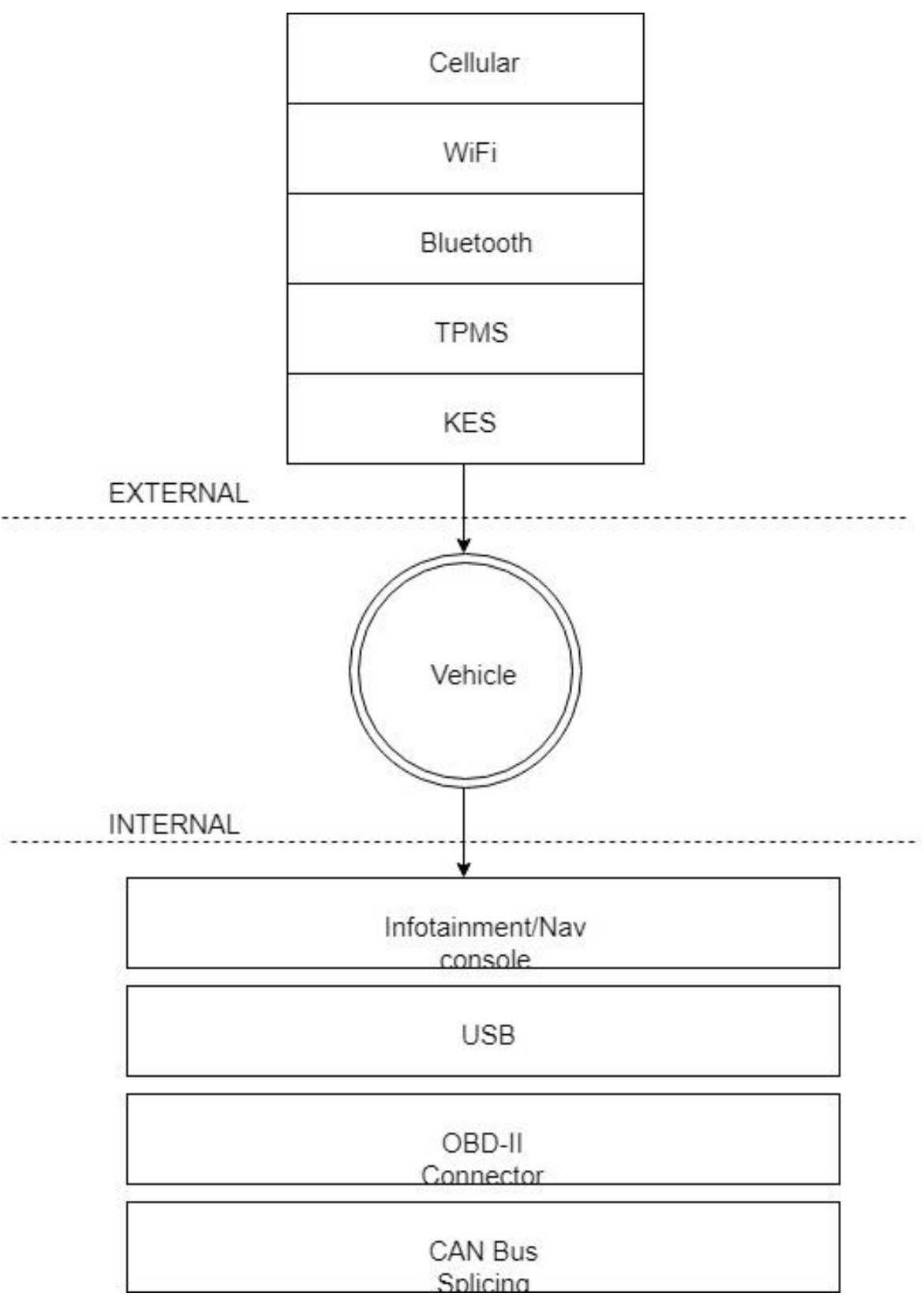

Fig.2 Connections

\section{RESULTS AND DISCUSSION}

CDMA and regular communication systems are tested of driverless and semi-driverless cars to test the suggested communication system performance. It was tested under various communication condition to confirm effective communication. One of the self-driving vehicles is programmed with the new CDMA communication system. The communication performance metrics are calculated of vehicles in two cases under same communication condition.

Conventional communication system need to be modified to adapt with new technology, such as self-driving vehicles. In other words, current security systems need to update to become more efficient with exiting communication systems. In addition, the proposed system plays important role in growing rate of packet that transmit between sender/ received as well as defensive these kids of VANETs. The proposed CDMA communication system can enhance transmission rate of autonomous and semiautonomous vehicles by increase packet delivery rate in its external

communication communications. It is mainly for dropping any request that target broadcasting packets. The role of the CDMA communication in providing slot time for sent/ received packet between cars in that zone.

Table. 1 Comparison between Existing and Proposed system

\begin{tabular}{|c|c|}
\hline $\begin{array}{c}\text { Existing system } \\
\text { communication } \\
\text { time(sec) }\end{array}$ & $\begin{array}{c}\text { Proposed system } \\
\text { communication time(sec) }\end{array}$ \\
\hline 3.5 & 1.2 \\
\hline 4.0 & 0.9 \\
\hline 2.9 & 2.0 \\
\hline 5.1 & 3.2 \\
\hline 4.8 & 2.2 \\
\hline
\end{tabular}




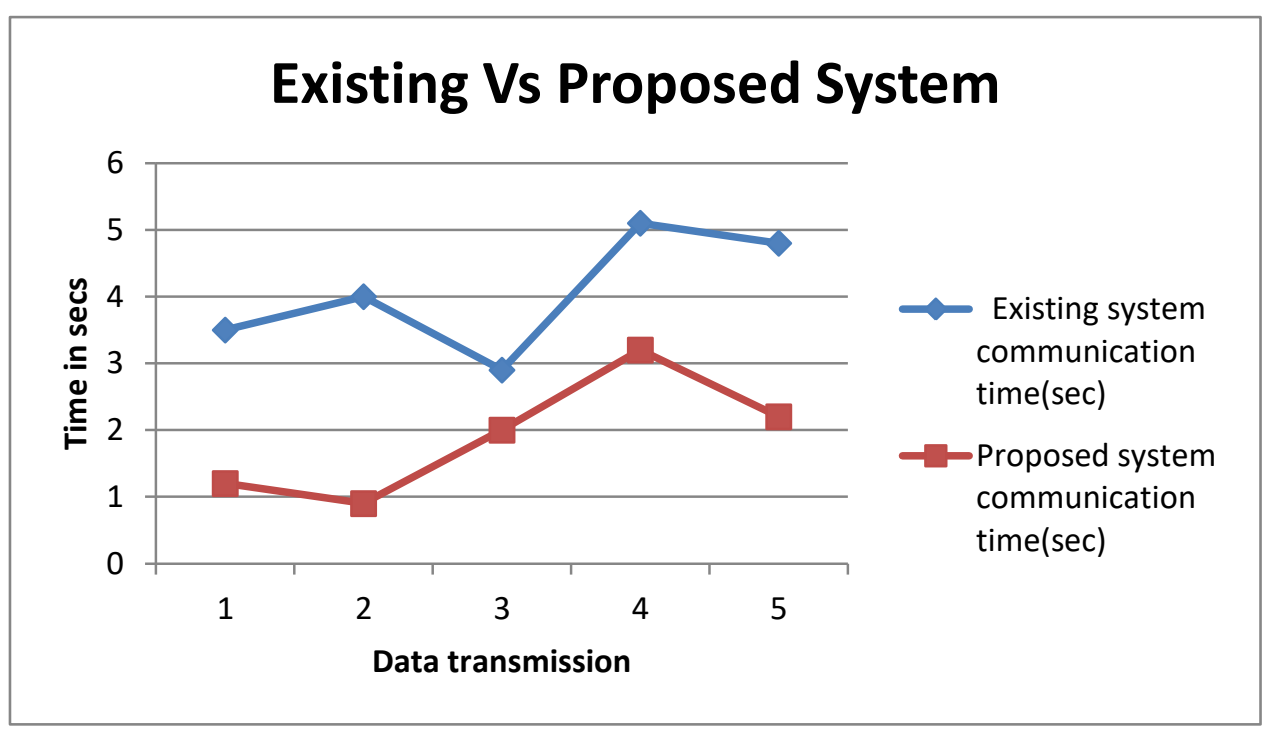

Fig. 3 Data transmission comparison for existing and proposed system

\section{CONCLUSION}

The self-driving vehicles has leading to several advantages in the world of automotive technology, and creating a new environment and new concepts aimed at human road security, because of most of the road accidents are caused by human. The most important part of selfdriving vehicles is how to introduce an efficient system to control all the required activities in this environment. Each vehicle has its unique identified code to be recognize from other vehicle and from the center. CDMA is introduced in this approach to generate a spreading code that is unique for each transmitter (vehicle). The spreading also code is used to extract the received data of the identify user. CDMA characterized the same frequency for high bandwidth, in addition of high security. The recommendation of future work in self-driving vehicles environment is to adapt the overall system via the huge environment of Internet of Thing (IoT).

The most external communications systems in autonomous vehicles including this approach have main limitation due to large differences between existing systems.

\section{REFERENCES}

1. J.J.M. Machado, P.M.-R. Gamarra, E.A.S.Marques, Lucas F.M. da Silva (2018)Improvement in impact strength of compositejoints for the automotive industry, CompositesPart B: Engineering, Volume 138, 1 April2018, Pages 243-255.

2. Xavier Ferràs-Hernández, ElisendaTarrats-Pons, NúriaArimany-Serrat (2017)Disruption in the automotive industry: ACambrian moment, Business Horizons,Volume 60, Issue 6, November-December2017, Pages 855-863.

3. J. Riznic (2017) CH1: Introduction to steamgenerators - from Heron of Alexandria tonuclear power plants: Brief history andliterature survey, Book chapter, SteamGenerators for Nuclear Power Plants, 2017,Pages 3-33.

4. Esa Kari Vakkilainen (2017) CH1: Principles ofSteam Generation, Book chapter, SteamGeneration from Biomass, 2017, Pages 1-17.

5. Karl Georg Høyer (2008) The history ofalternative fuels in transportation: The case ofelectric and hybrid cars, Utilities Policy,Volume 16, Issue 2, June 2008, Pages 63-71.

6. Marc Dijk (2011) Technological frames of carengines, Technology in Society, Volume 3,Issues 1-2, February-May 2011, Pages 165-180.

7. ChristoforosMavrelos, GerasimosTheotokatos(2018) Numerical investigation of a premixedcombustion large marine two-stroke dual fuelengine for optimising engine settings viaparametric runs, Energy Conversion andManagement, Volume 160, 15 March 2018,Pages 48 59.

8. Yong Qian, Liang Yu, Zilong Li, YahuiZhang,Xingcai Lu (2018) A new methodology fordiesel surrogate fuel formulation: Bridgingfuel fundamental properties and real enginecombustion characteristics, Energy, Volume148, 1 April 2018, Pages 424-447.

9. TakuTsujimura, Yasumasa Suzuki (2017) Theutilization of hydrogen in hydrogen/dieseldual fuel engine, International Journal ofHydrogen Energy, Volume 42, Issue 19, 11May 2017, Pages 14019-14029.

10. Steven Gray (2017) Fueling mobility: coal and .Britain's naval power, c. 1870-1914, Journalof Historical Geography, Volume 58, October2017, Pages 92-103.

11. John Warner (2015) Chapter 2: History ofVehicle Electrification, Book chapter, TheHandbook of Lithium-Ion Battery PackDesign, 2015, Pages 9-21.

12. Noriko HikosakaBehling (2013) Chapter 3:History of Alkaline Fuel Cells, Book chapter,Fuel Cells, 2013, Pages 37-51.

13. Diya Thomas, Binsu C. Kovoor (2018) AGenetic Algorithm Approach to AutonomousSmart Vehicle Parking system, Procedia.

14. Jamal Aslam, A Survey On Routing Mechanism In Vehicle To Vehicle Communication Using Vanet, "International Innovative Research Journal of Engineering and Technology", 3(2), 2017. 